This information is current as of April 25, 2023. 


\title{
Noncontrast Head CT in Children: National Variation in Radiation Dose Indices in the United States
}

\author{
DG. Sadigh, (D) N. Kadom, DP. Karthik, D. Sengupta, (D). J. Strauss, DD. Frush, and (D)E. Applegate
} $=\square$
$\square$

\begin{abstract}
BACKGROUND AND PURPOSE: Radiologists should manage the radiation dose for pediatric patients to maintain reasonable diagnostic confidence. We assessed the variation in estimated radiation dose indices for pediatric noncontrast head CT in the United States.
\end{abstract}

MATERIALS AND METHODS: Radiation dose indices for single-phase noncontrast head CT examinations in patients 18 years of age and younger were retrospectively reviewed between July 2011 and June 2016 using the American College of Radiology CT Dose Index Registry. We used the reported volume CT dose index stratified by patient demographics and imaging facility characteristics.

RESULTS: The registry included 295,296 single-phase pediatric noncontrast head CT studies from 1571 facilities (56\% in male patients and $53 \%$ in children older than 10 years of age). The median volume CT dose index was $33 \mathrm{mGy}$ (interquartile range $=22-47 \mathrm{mGy}$ ). The volume CT dose index increased as age increased. The volume CT dose index was lower in children's hospitals (median, 26 mGy) versus academic hospitals (median, $32 \mathrm{mGy}$ ) and community hospitals (median, $40 \mathrm{mGy}$ ). There was a lower volume CT dose index in level I and II trauma centers (median, 27 and 32 mGy, respectively) versus nontrauma centers (median, 40 mGy) and facilities in metropolitan locations (median, $30 \mathrm{mGy}$ ) versus those in suburban and rural locations (median, $41 \mathrm{mGy}$ ).

CONCLUSIONS: Considerable variation in the radiation dose index for pediatric head CT exists. Median dose indices and practice variations at pediatric facilities were both lower compared with other practice settings. Decreasing dose variability through proper management of CT parameters in pediatric populations using benchmarks generated by data from registries can potentially decrease population exposure to ionizing radiation.

ABBREVIATIONS: $\mathrm{CTDI}_{\mathrm{vol}}=\mathrm{CT}$ dose index volume; $\mathrm{DIR}=$ Dose Index Registry; $\mathrm{DLP}=$ dose-length product; $\mathrm{IQR}=$ interquartile range

l: 2011, eighty-five million CT scans were performed in the United States; approximately $5 \%-11 \%$ of these scans were performed on children. ${ }^{1,2}$ A more recent study using the American College of Radiology Dose Index Registry (DIR) reported that approximately $6 \%$ of CT scans performed in participating facilities the United States were performed on children. ${ }^{3}$ CT scans

Received February 11, 2018; accepted after revision May 18.

From the Department of Radiology and Imaging Sciences (G.S., N.K.,), Emory University School of Medicine, Atlanta, Georgia; American College of Radiology (P.K. D.S.), Reston, Virginia; Department of Radiology (K.J.S.), University of Cincinnati School of Medicine, Cincinnati, Ohio; Department of Radiology (D.F.), Duke University Medical Center, Durham, North Carolina; and Department of Radiology (K.E.A.), University of Kentucky, Lexington, Kentucky.

Abstract previously presented as a poster at: Annual Meeting of the American College of Radiology, May 21-25, 2017; Washington, DC.

Please address correspondence to Gelareh Sadigh, MD, Department of Radiology and Imaging Sciences, Emory University School of Medicine, 1364 Clifton Rd NE, Atlanta, GA 30322; email: gsadigh@emory.edu; @GelarehSadigh

Indicates article with supplemental on-line table.

Indicates article with supplemental on-line photo.

http://dx.doi.org/10.3174/ajnr.A5719 alone accounted for about $50 \%$ and $75 \%$ of the radiation doses in adults and in children, respectively. ${ }^{4}$ The most frequently imaged pediatric body part was the head. ${ }^{3}$ The most common indication was trauma, followed by headache, convulsions, and syncope. ${ }^{5,6}$ In the past 2 decades, there has been an increase in the use of CT scans in pediatric patients in emergency departments, which includes both first-time and repeat head CT scans. ${ }^{6-8}$

Many attempts have been made to quantify risks associated with ionizing radiation use in medical imaging. Some studies have suggested that the radiation dose from pediatric head CT scans may increase the risk of developing leukemia or other solid tumors. ${ }^{9-12}$ Another study has suggested that the radiation dose to the lens of the eye from head and neck CT scans may increase the risk of cataracts. ${ }^{13}$ A recent cohort study of nuclear medicine technologists suggested a higher risk of cataracts from their occupational radiation exposures. ${ }^{14}$ Children are more radiosensitive than adults and, therefore, have a higher risk of developing cataracts. ${ }^{15}$ They are also more likely to be affected by cancers such as leukemia, brain, breast, skin, and thyroid cancer as well as cardiovascular diseases after high doses of 
radiation from therapies. Interpretation of some of the literature is controversial as they associate cancer risks with ionizing radiation on the basis of myriad assumptions. ${ }^{16-18}$ Nevertheless, pediatric patients do have a longer expected lifetime during which diseases and radiation-related complications might occur, which may or may not be linked to ionizing radiation.

The As Low As Reasonably Achievable principle guides radiologists to use the lowest radiation dose on any given imaging examination that will answer the clinical question, yet preserve the radiologist's ability to make a diagnosis. This diagnostic capability can be influenced by many practical issues, including patient factors such as motion or the degree of medical illness/complexity, technical factors, and the level of training and experience of the interpreting radiologist. Therefore, there is an expected wide variability of imaging practices within a framework of best practice. The radiation dose from head CT and its variation in the pediatric population has had a limited focus, with small institutional studies or surveys ${ }^{19-22}$ each demonstrating substantial variation in radiation dose. However, the optimal current practices and the magnitude of variation in the radiation dose delivered by pediatric noncontrast head CT have not been assessed at the national level. Participation in dose registries allows those providers who care for children the opportunity to compare their performance against published national benchmarks. ${ }^{23}$

The purpose of this study was to assess variations in estimated radiation dose indices for pediatric noncontrast head CT in the United States using the American College of Radiology DIR.

\section{MATERIALS AND METHODS \\ Study Design}

This was a retrospective analysis of pediatric noncontrast head CT examinations in the American College of Radiology DIR from July 1, 2011, through June 30,2016. Because this study qualified as "nonhuman subject" research, institutional review board oversight was not required by our institution.

\section{Dose Index Registry}

The DIR launched in mid-2011 by the American College of Radiology is a national data base of dose indices associated with CT scans, which allows facilities to compare their dose indices with those at other facilities as well as other population and geographic denominations and to improve their practices when appropriate. ${ }^{24,25}$ Given that different facilities may use different examination names for the same imaging test, when a facility submits data to the DIR, every CT examination name used at that facility is mapped to a standardized list of examination names used by the DIR, using the RadLex Playbook identifiers (https://www.rsna.org/RadLex_ Playbook.aspx). ${ }^{24}$ All of the data presented in this study came from facilities that submit their data to the DIR data base.

\section{Study Setting and Population}

We included noncontrast head CT examinations performed in patients 18 years of age or younger during the study period. Multiphase examinations (ie, with and without contrast examinations) were excluded to avoid overestimating the radiation dose index. Furthermore, these examinations are not routine in pediatric head CT.

\section{Study Protocol}

We used the following examination names submitted by participating facilities mapped in the DIR to define noncontrast head CT examinations: "CT BRN WO IVCON," "CT HEAD BRN WO IVCON," "CT HEAD BRN DUAL ENG CT WO IVCON," "CT HEAD MLTPL AREA SM BDY REG WO IVCON," "CT PEDS HEAD WO IVCON," "CT HEAD TRAUMA WO IVCON," "CT PEDS HEAD BRN WO IVCON," "CT HEAD WO IVCON." These examinations included noncontrast head CT using routine or low-dose protocols. Of note, institutions may name CT examinations differently, and the name under which an examination was listed in the DIR may not reflect whether a low-dose protocol was actually used.

The 2 primary CT dose indices, which can be used to calculate estimates of the radiation dose to the patient during head scans, were analyzed in this study. The first, CT dose index volume $\left(\mathrm{CTDI}_{\mathrm{vol}}\right)$ is an indication of the average absorbed radiation within the scan volume for a standardized cylindric CTDI phantom. ${ }^{26}$ In children, this is made of Plexiglas (https://www. plexiglas-shop.com/shopselect.htm) with a diameter of $16 \mathrm{~cm}$. The second, dose-length product (DLP), is the product of $\mathrm{CTDI}_{\mathrm{vol}}$ and scan length along the z-axis of the patient, which estimates the total energy delivered to the CTDI phantom during the examination. The scan length can be estimated by dividing the DLP by the CTDI ${ }_{\text {vol }} \cdot{ }^{26}$

We obtained additional information including patient demographics (age, sex), characteristics of the imaging facility, and the year the examination was performed using the DIR data base. Age groups were defined on the basis of previous work ${ }^{5}$ and were as follows: $0-2,3-6,7-10,11-14$, and 15-18 years of age. Imaging facility characteristics included hospital type (academic, community hospital, multispecialty clinic, freestanding center, children's hospital, and other), trauma designation (I, II, III, IV, and not applicable), facility location (metropolitan, suburban, and rural), and census regions (Northeast, Midwest, South, and West) based on the US Census Bureau categorization. ${ }^{27}$ Because facilities can designate only a single hospital type, facilities designated as academic and community were further assigned as pediatric if they used "children" in the name or if they performed at least $75 \%$ of the total examinations on children 18 years of age or younger ${ }^{5}$ as defined by the DIR.

\section{Data Analysis}

We used summary statistics to describe the patient and hospital characteristics, including number and frequency. The CTDI ${ }_{\text {vol }}$ and DLPs were reported as median and interquartile range (IQR). The median CTDI $_{\mathrm{vol}}$ was compared among the categories for each variable using the Kruskal-Wallis test. We further compared the median $\mathrm{CTDI}_{\mathrm{vol}}$ among facility types adjusting on the basis of patient age and sex using the ANOVA test. The data analysis for this article was generated using SAS software (SAS Institute, Cary, North Carolina). $P$ values $\leq .05$ were considered statistically significant. The results were displayed graphically using 5 pediatric age categories $(0-2,3-6,7-10,11-14$, and $15-18$ years of age) that the American College of Radiology DIR provides to all enrolled facilities.

AJNR Am J Neuroradiol 39:1400-05 Aug 2018 www.ajnr.org 1401 


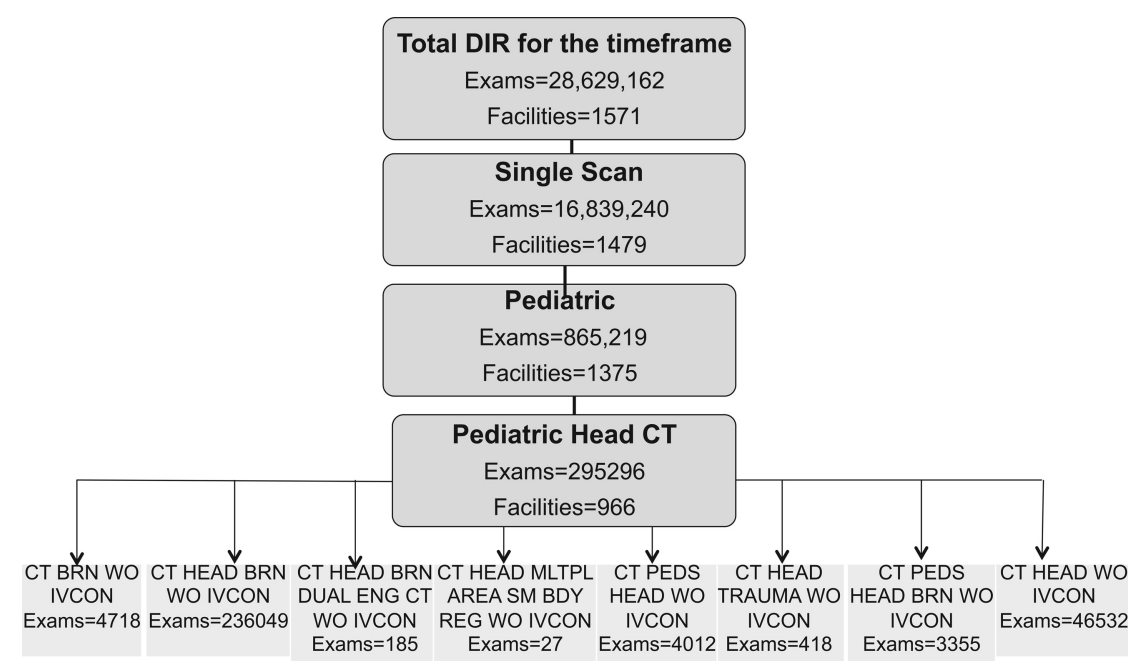

FIG. Head CT examinations included in the study cohort from the DIR. group, sex, and other facility characteristics, the mean CTDI ${ }_{\mathrm{vol}}$ was statistically significantly different among facility types $(P<.0001)$, lower age groups of $0-2$ and 3-6 years did not have any clinically significant variations in their mean $\mathrm{CTDI}_{\mathrm{vol}}$ among facility types (On-line Figure). Community hospitals, which had the largest proportion of examinations, had a median CTDI $_{\mathrm{vol}}$ of $40 \mathrm{mGy}$ $(\mathrm{IQR}=27-52 \mathrm{mGy})$. The variations in the median $\mathrm{CTDI}_{\mathrm{vol}}$ for examinations were also statistically significant among facility trauma designations $(P<.0001)$. Trauma levels I and II had the lowest median $\mathrm{CTDI}_{\mathrm{vol}}(27 \mathrm{mGy}$ for level I and $32 \mathrm{mGy}$ for level II) compared with other hospitals. The facilities with average monthly examinations of $\leq 100 \mathrm{had}$

\section{RESULTS}

\section{Patient Population}

Of the 28,629,162 CT examinations within the DIR data base, $59 \%(n=16,839,240)$ were performed as a single scan, and $5 \%$ of these single scans $(n=865,219)$ were obtained in patients 18 years of age or younger. Of these pediatric single scans, $34 \%$ ( $n=$ 295,296) were noncontrast head CT scans (Figure).

Examinations in male patients represented 56\% $(n=165,482)$ of examinations (On-line Table). The distribution of head CTs across patients in pediatric age groups was as follows: $21 \%(n=$ $62,933)$ in $0-2$ years, $13 \%(n=38,144)$ in $3-6$ years, $13 \%(n=$ $38,847)$ in $7-10$ years, $20 \%(n=58,091)$ in $11-14$ years, and $33 \%$ $(n=97,281)$ in $15-18$ years.

\section{Hospitals}

There were a total of 1571 facilities in the DIR during the study period, and 966 facilities (61\%) performed pediatric single noncontrast head CT scans (Figure). Most of these facilities (55\%; $533 / 966)$ were community hospitals and 24\% (226/966) were designated as trauma levels I and II.

While only $42 \%$ (404/966) of facilities performing pediatric single noncontrast head CT scans were in metropolitan areas, $65.7 \%(n=194,155)$ of these examinations were performed in these facilities. Furthermore, 32\% of the facilities (304/966) were in the Southern regions and $43 \%(n=126,874)$ of examinations were performed in this area. More detailed information on patient demographics and characteristics of the imaging facilities are listed in the On-line Table.

\section{Radiation Dose Indices}

Across all pediatric single noncontrast head CT examinations, the median $\mathrm{CTDI}_{\mathrm{vol}}$ was $33 \mathrm{mGy}(\mathrm{IQR}=22-47 \mathrm{mGy})$, with approximately $107 \%$ difference between the 25 th and 75 th percentiles. The median $\mathrm{CTDI}_{\mathrm{vol}}$ was higher for older age groups, as expected (On-line Table). The variations in the median CTDI ${ }_{\text {vol }}$ for examinations were statistically significant among facility types $(P<$ .0001). The pediatric facilities had a lower median CTDI ${ }_{\mathrm{vol}}(26$ mGy, IQR = 19-33 mGy) compared with other facility types. Even though statistical tests showed that when controlling for age higher median $\mathrm{CTDI}_{\mathrm{vol}}$ compared with facilities with $>100$ examinations (On-line Table).

For all pediatric single noncontrast head CT examinations, the median DLP was $543 \mathrm{mGy} \times \mathrm{cm}(\mathrm{IQR}=357-758 \mathrm{mGy} \times \mathrm{cm})$, with an approximately $112 \%$ difference between the 25 th and 75th percentiles. This finding corresponds to a median scan length of $16.5 \mathrm{~cm}$ along the $\mathrm{z}$-axis of the patient for a pediatric head examination regardless of age. Accordingly, the median scan length for the $0-2$ years of age group was $14.7 \mathrm{~cm}$ compared with a range of $16.2-16.7 \mathrm{~cm}$ for all patients older than 2 years of age.

\section{DISCUSSION}

The results of the current national study of radiation dose indices for pediatric single-phase noncontrast head CT showed variations in radiation dose estimates as measured by CTDI $_{\text {vol }}$ and DLP, with approximately $107 \%$ and $112 \%$ difference between the 25 th and 75th percentiles, respectively. As expected, the CTDI ${ }_{\mathrm{vol}}$ increased with patient age. The median dose indices were lower at pediatric facilities compared with other facility types and lower in metropolitan areas compared with suburban and rural facilities.

Our results are similar to findings from prior studies (Table 1) showing practice variations among imaging facilities. Prior studies have reported that the dose indices of pediatric hospitals were less than those in general hospitals ${ }^{21}$ or trauma centers. ${ }^{19}$ A national survey of US hospitals using the American Hospital Association 2010 annual survey data base showed that $82 \%$ of hospitals reported doses of $<40 \mathrm{mGy} .{ }^{21}$ Practice variations were found in a survey of trauma centers in the state of Washington, which showed a large proportion of level III-V trauma facilities tended to have higher milliampere-second values due to late adoption of dose-reduction strategies, resulting in larger median CTDI $_{\text {vol }}$ values. ${ }^{20}$ Our results showed that facilities in metropolitan areas had lower median CTDI $_{\mathrm{vol}}$ compared with urban and rural locations $(P<.0001)$ possibly due to the poorer quality imaging equipment (eg, low-resolution equipment) in the smaller, rural facilities, ${ }^{28}$ which requires a greater patient dose to produce acceptable images. Imaging facilities in the metropolitan areas may also be academic centers, which continually try to follow benchmarks 
Table 1: Studies of radiation dose indices for pediatric noncontrast head CT

\begin{tabular}{|c|c|c|c|}
\hline Study & $\begin{array}{l}\text { Publication } \\
\text { Year }\end{array}$ & Type & Findings \\
\hline Kanal et $\mathrm{al}^{21}$ & 2015 & National survey & Pediatric hospitals had lower dose indices compared with general hospitals \\
\hline Kanal et $\mathrm{al}^{20}$ & 2011 & Washington State survey & Level I-II trauma centers had lower dose indices compared with Levels III-V \\
\hline King et $\mathrm{al}^{19}$ & 2009 & Bi-institutional studies & $\begin{array}{l}\text { Regional pediatric hospitals had lower dose indices compared with trauma } \\
\text { centers }\end{array}$ \\
\hline Current study & 2018 & National study of ACR DIR & $\begin{array}{l}\text { Pediatric hospitals had lower dose indices compared with other facility types } \\
\text { Level I-II trauma centers had lower dose indices compared with level III-IV } \\
\text { trauma centers or nontrauma hospitals } \\
\text { Facilities in metropolitan areas had lower dose indices compared with urban } \\
\text { and rural locations }\end{array}$ \\
\hline
\end{tabular}

Note:-ACR indicates American College of Radiology.

\section{Table 2: Suggested dose-reduction strategies}

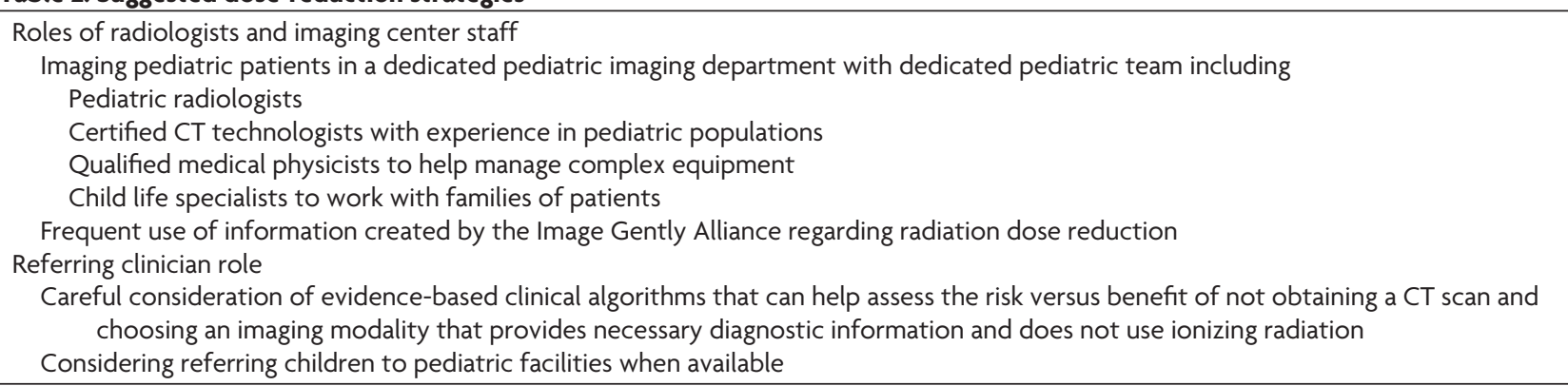

and compete with other centers. This effort can be facilitated by national, educational, or institutional campaigns. ${ }^{22}$

As expected, our results show that both $\mathrm{CTDI}_{\mathrm{vol}}$ and DLP increase with age. However, younger patients are expected to experience increases in DLP due to both an increase in CTDI $_{\mathrm{vol}}$ and scan length, given increasing head sizes. The scan lengths were 14.7 and $16.4 \mathrm{~cm}$ for the 0 - to 2-year and 3- to 6-year age groups, respectively. The scan length in patients younger than 6 years of age being only slightly shorter than that in adult-sized heads, suggests that the total energy deposited in the small pediatric patients' heads could possibly be reduced further by more careful control of the beginning and end of the chosen scan length by the operator. In addition, the smaller the child, the more likely that radiosensitive organs are included within the imaging area of interest. ${ }^{29}$

Multiple causes result in a wide range of patient radiation doses, including variability of equipment makes and models, ${ }^{30,31}$ patient positioning, ${ }^{32}$ and anatomic coverage (eg, scan length versus length of patient anatomy of interest). ${ }^{33}$ All factors controlled by the technologist (eg, voltage, tube current, rotation time, pitch, scan length, bow-tie filter, and so forth) can affect the range of patient doses when not set properly. ${ }^{34}$

Imaging pediatric patients with a dedicated pediatric team that includes pediatric radiologists, certified CT technologists with experience in the pediatric population, qualified medical physicists to help manage complex equipment, and child life specialists to work with families of patients in a dedicated pediatric imaging department may result in improved pediatric CT protocols that reduce the patient dose while maintaining image quality (Table 2). ${ }^{35}$ The Image Gently Alliance has created information to help facilities achieve these types of goals. ${ }^{36-38}$

While radiologists, medical physicists, and CT technologists directly manage CT imaging techniques, clinicians can influence the patients' radiation doses by careful consideration of evidencebased clinical algorithms, which can help assess the risk versus benefit of not obtaining a CT examination and choosing an imaging technique that provides the necessary diagnostic information and does not use ionizing radiation. Clinicians may also consider referring children to pediatric facilities when available (Table 2). ${ }^{5,39}$

Our study has several limitations. First, both CT dose indices for the head and CTDI ${ }_{\mathrm{vol}}$ and DLP stored by the DIR are estimates of the dose to a standard plastic phantom as opposed to the patient. However, these are the only $2 \mathrm{CT}$ dose indices available until the American Association for Physicists in Medicine finishes its development of a size-specific dose estimate to serve the same purpose for the head as the size-specific dose estimate currently serves for the trunk. ${ }^{26}$ When the head-size-specific dose estimate is available, it will be expressed as a function of the size of the head, which correlates better to patient dose than the age of the patient. ${ }^{40}$ Second, because participation in the DIR is voluntary, there may be a selection bias in our study population. ${ }^{5}$ Facilities involved in the DIR may be more likely to audit their practices and follow dose-reduction protocols and dose-modification techniques; therefore, our dose-estimate results may be an underestimate of both CT doses and the variation in current CT practices. ${ }^{5}$ Third, data collected in the early years of the DIR (2011 launch) may contain some systematic errors that were reduced or eliminated with more experience. Fourth, institutions may name CT examinations differently, which can be associated with inconsistent or incorrect mapping to the standardized list of examinations used by the DIR. ${ }^{41}$ Furthermore, the name under which an examination was listed in the DIR may not reflect whether a low-dose protocol was used. It is possible that pediatric facilities perform low-dose head CT examinations more commonly for evaluation of ventricular shunt catheters; therefore, their median CTDI $_{\mathrm{vol}}$ is lower compared with nonpediatric facilities. Finally, facilities continually change their protocols with time, especially as more 
attention is given to the importance of checking doses and having appropriate protocols.

\section{CONCLUSIONS}

Practice variations in the radiation dose index for pediatric head CT exist. Less variation occurred in pediatric compared with adult facilities and in metropolitan areas compared with suburban/rural facilities. Decreasing dose variability through proper management of CT parameters in pediatric populations using benchmarks generated by data from registries may help decrease population exposure to ionizing radiation.

Disclosures: Keith J. Strauss_UNRELATED: Consultancy: Phillips Medical Systems of North America, Comments: assisted with assignments as a medical physics consultant as requested by the entity.

\section{REFERENCES}

1. Miglioretti DL, Johnson E, Williams A, et al. The use of computed tomography in pediatrics and the associated radiation exposure and estimated cancer risk. JAMA Pediatr 2013;167:700-07 CrossRef Medline

2. Mettler FA Jr, Wiest PW, Locken JA, et al. CT scanning: patterns of use and dose. J Radiol Prot 2000;20:353-59 CrossRef Medline

3. Wildman-Tobriner B, Strauss KJ, Bhargavan-Chatfield M, et al. Using the American College of Radiology Dose Index Registry to evaluate practice patterns and radiation dose estimates of pediatric body CT. AJR Am J Roentgenol 2018;210:641-47 CrossRef Medline

4. Mettler FA Jr, Thomadsen BR, Bhargavan M, et al. Medical radiation exposure in the U.S. in 2006: preliminary results. Health Phys 2008; 95:502-07 CrossRef Medline

5. Marin JR, Sengupta D, Bhargavan-Chatfield M, et al. Variation in pediatric cervical spine computed tomography radiation dose index. Acad Emerg Med 2015;22:1499-505 CrossRef Medline

6. Larson DB, Johnson LW, Schnell BM, et al. Rising use of CT in child visits to the emergency department in the United States, 1995-2008. Radiology 2011;259:793-801 CrossRef Medline

7. Hollingworth W, Vavilala MS, Jarvik JG, et al. The use of repeated head computed tomography in pediatric blunt head trauma: factors predicting new and worsening brain injury. Pediatr Crit Care Med 2007;8:348-56; CEU quiz 357 CrossRef Medline

8. Statler KD, Keenan H. How many are too many? Repeat head computed tomography after traumatic brain injury. Pediatr Crit Care Med 2007;8:402-03 CrossRef Medline

9. Pearce MS, Salotti JA, Little MP, et al. Radiation exposure from CT scans in childhood and subsequent risk of leukaemia and brain tumours: a retrospective cohort study. Lancet 2012;380:499-505 CrossRef Medline

10. Boice JD Jr. Radiation epidemiology and recent paediatric computed tomography studies. Ann ICRP 2015;44:236-48 CrossRef Medline

11. The National Academics. Beir VII: Health Risks from Exposure to Low Levels of Ionizing Radiation. https://www.nap.edu/read/11340/ chapter/1. Accessed June 10, 2018

12. Mathews JD, Forsythe AV, Brady Z, et al. Cancer risk in $\mathbf{6 8 0 , 0 0 0}$ people exposed to computed tomography scans in childhood or adolescence: data linkage study of 11 million Australians. $B M J$ 2013;346:f2360 CrossRef Medline

13. Yuan MK, Tsai DC, Chang SC, et al. The risk of cataract associated with repeated head and neck CT studies: a nationwide population-based study. AJR Am J Roentgenol 2013;201:626-30 CrossRef Medline

14. Bernier MO, Journy N, Villoing D, et al. Cataract risk in a cohort of U.S. radiologic technologists performing nuclear medicine procedures. Radiology 2018;286:592-601 CrossRef Medline

15. Nakashima E, Neriishi K, Minamoto A. A reanalysis of atomic-bomb cataract data, 2000-2002: a threshold analysis. Health Phys 2006;90: 154-60 CrossRef Medline

16. Gardavaud F, Luciani A, Rahmouni A. CT scans in childhood and risk of leukaemia and brain tumours. Lancet 2012;380:1735; author reply 1736-37 CrossRef Medline

17. Zopf DA, Green GE. CT scans in childhood and risk of leukaemia and brain tumours. Lancet 2012;380:1735-36; author reply 1736-37

18. United States Nuclear Regulatory Commission. Radiation Exposure and Cancer. https://www.nrc.gov/about-nrc/radiation/health-effects/ rad-exposure-cancer.html. Accessed February 4, 2018

19. King MA, Kanal KM, Relyea-Chew A, et al. Radiation exposure from pediatric head CT: a bi-institutional study. Pediatr Radiol 2009;39: 1059-65 CrossRef Medline

20. Kanal KM, Vavilala MS, Raelson C, et al. Variation in pediatric head CT imaging protocols in Washington state. J Am Coll Radiol 2011;8: 242-50 CrossRef Medline

21. Kanal KM, Graves JM, Vavilala MS, et al. Variation in CT pediatric head examination radiation dose: results from a national survey. AJR Am J Roentgenol 2015;204:W293-301 CrossRef Medline

22. Graves JM, Kanal KM, Rivara FP, et al. Dose reduction efforts for pediatric head CT imaging in Washington State trauma centers: follow-up survey results. J Am Coll Radiol 2014;11:161-68 e3 CrossRef Medline

23. Kanal KM, Butler PF, Sengupta D, et al. U.S. diagnostic reference levels and achievable doses for 10 adult CT examinations. Radiology 2017;284:120-33 CrossRef Medline

24. American College of Radiology. Dose Index Registry. http://www.acr. org/Quality-Safety/National-Radiology-Data-Registry/DoseIndex-Registry. Accessed October 13, 2017

25. Morin RL, Coombs LP, Chatfield MB. ACR Dose Index Registry. J Am Coll Radiol 2011;8:288-91 CrossRef Medline

26. American Association of Physicist in Medicine. The Measurement, Reporting, and Management of Radiation Dose in CT. Report of AAPM Task Group 23 of the Diagnostic Imaging Council CT Committee. January 2008. https://www.aapm.org/pubs/reports/RPT_ 96.pdf. Accessed January 25, 2018

27. U.S. Census Bureau. Census Regions and Divisions of the United States. http://www2.census.gov/geo/pdfs/maps-data/maps/reference/ us_regdiv.pdf. Accessed October 13, 2017

28. Ginde AA, Foianini A, Renner DM, et al. Availability and quality of computed tomography and magnetic resonance imaging equipment in U.S. emergency departments. Acad Emerg Med 2008;15: 780-83 CrossRef Medline

29. Image Gently. Image Gently launches the Think-A-head Campaign. https://rpop.iaea.org/RPOP/RPoP/Content/News/10-Image_Gently. htm. Accessed October 19, 2017

30. Jaffe TA, Yoshizumi TT, Toncheva G, et al. Radiation dose for body CT protocols: variability of scanners at one institution. AJR Am J Roentgenol 2009;193:1141-47 CrossRef Medline

31. Mayo-Smith WW, Hara AK, Mahesh M, et al. How I do it: managing radiation dose in CT. Radiology 2014;273:657-72 CrossRef Medline

32. Brink M, de Lange F, Oostveen LJ, et al. Arm raising at exposurecontrolled multidetector trauma CT of thoracoabdominal region: higher image quality, lower radiation dose. Radiology 2008;249: 661-70 CrossRef Medline

33. Campbell J, Kalra MK, Rizzo S, et al. Scanning beyond anatomic limits of the thorax in chest CT: findings, radiation dose, and automatic tube current modulation. AJR Am J Roentgenol 2005;185: 1525-30 CrossRef Medline

34. Harri PA, Moreno CC, Nelson RC, et al. Variability of MDCT dose due to technologist performance: impact of posteroanterior versus anteroposterior localizer image and table height with use of automated tube current modulation. AJR Am J Roentgenol 2014;203: 377-86 CrossRef Medline

35. Borders HL, Barnes CL, Parks DC, et al. Use of a dedicated pediatric CT imaging service associated with decreased patient radiation dose. J Am Coll Radiol 2012;9:340-43 CrossRef Medline

36. Goske MJ, Applegate KE, Boylan J, et al. The 'Image Gently' 
campaign: increasing CT radiation dose awareness through a national education and awareness program. Pediatr Radiol 2008;38: 265-69 CrossRef Medline

37. Goske MJ, Applegate KE, Boylan J, et al. The Image Gently campaign: working together to change practice. AJR Am J Roentgenol 2008;190: 273-74 CrossRef Medline

38. Strauss KJ, Goske MJ, Kaste SC, et al. Image Gently: ten steps you can take to optimize image quality and lower CT dose for pediatric patients. AJR Am J Roentgenol 2010;194:868-73 CrossRef Medline
39. American College of Radiology. Appropriateness Criteria. https:// acsearch.acr.org/list. Accessed January 25, 2018

40. Kleinman PL, Strauss KJ, Zurakowski D, et al. Patient size measured on CT images as a function of age at a tertiary care children's hospital. AJR Am J Roentgenol 2010;194:1611-19 CrossRef Medline

41. Bohl M. Dose Index Registry Exam Name Mapping Review Made Easy. https://wwwlinkedincom/pulse/dose-index-registry-examname-mapping-review-made-easy-michael-bohl/ 2017. Accessed April 13, 2018 